\title{
Return of the WIMP: Missing energy signals and the Galactic Center excess
}

\author{
Marcela Carena, ${ }^{1,2, *}$ James Osborne $\odot,{ }^{3, \dagger}$ Nausheen R. Shah, ${ }^{3, *}$ and Carlos E. M. Wagner ${ }^{2,4, \S}$ \\ ${ }^{1}$ Fermi National Accelerator Laboratory, P. O. Box 500, Batavia, Illinois 60510, USA \\ ${ }^{2}$ Enrico Fermi Institute and Kavli Institute for Cosmological Physics, University of Chicago, \\ Chicago, Illinois 60637, USA \\ ${ }^{3}$ Department of Physics \& Astronomy, Wayne State University, Detroit, Michigan 48201, USA \\ ${ }^{4}$ HEP Division, Argonne National Laboratory, 9700 Cass Ave., Argonne, Illinois 60439, USA
}

(Received 19 June 2019; published 3 September 2019)

\begin{abstract}
In a recent work, we emphasized that an excess in trilepton events plus missing energy observed by the ATLAS experiment at the LHC could be interpreted as a signal of low-energy supersymmetry. In such a scenario the lightest neutralino mass is approximately $m_{\chi} \simeq 60 \mathrm{GeV}$ and the direct dark matter detection cross section is naturally below the current bound. In this work we present simple extensions of this scenario that lead to an explanation of the gamma-ray excess at the center of the Galaxy observed by FermiLAT, as well as the antiproton excess observed by AMS-02. These extensions include the addition of a small $C P$-violating phase in the neutralino sector or the addition of a light $C P$-odd Higgs scalar. Our study is of special relevance in view of a recent analysis that casts doubt on the previously accepted preference for millisecond pulsars as the origin of the Galactic Center excess.
\end{abstract}

DOI: 10.1103/PhysRevD.100.055002

\section{INTRODUCTION}

Low-energy supersymmetry is undergoing heavy scrutiny from a large number of experimental probes, including missing energy signatures at the LHC, and direct and indirect dark matter (DM) searches. Currently, collider searches for low-energy supersymmetry (SUSY) have led to no conclusive evidence of its existence at the weak scale. Strongly interacting supersymmetric particles are constrained by LHC experiments to be above a scale of the order of a TeV [1-6], whereas bounds on weakly interacting particles are far less severe, and are of the order of a few hundred $\mathrm{GeV}$.

In a previous article [7] we interpreted a recently observed excess in trilepton plus missing energy events [8] in terms of low-energy SUSY. The pertinent LHC analysis was performed by the ATLAS Collaboration using a newly developed recursive jigsaw reconstruction (RJR) method $[9,10]$, which is well suited to study compressed spectra, and is sensitive to regions of parameters that may lead to no apparent signal events in conventional searches

\footnotetext{
carena@fnal.gov

†jaosborne@wayne.edu

nausheen.shah@wayne.edu

§cwagner@anl.gov
}

Published by the American Physical Society under the terms of the Creative Commons Attribution 4.0 International license. Further distribution of this work must maintain attribution to the author(s) and the published article's title, journal citation, and DOI. Funded by SCOAP.
$[11,12]$. The reported excess was further supported by analyses performed by the GAMBIT Collaboration, which argued that since the bounds from conventional searches were obtained by simplified scenarios, they may not accurately capture the properties of signals obtained in more realistic models $[13,14]$. The preferred region of masses to explain the RJR excess was found to be for a mostly bino-like neutralino with a mass of about $60 \mathrm{GeV}$, and a mostly wino-like neutralino with a mass of about $160 \mathrm{GeV}$. Obtaining the proper DM relic density lead to the preference for Higgsinos with masses below $500 \mathrm{GeV}$, and values of $\tan \beta \gtrsim 10$. This region of parameters leads to a sizable contribution to the muon anomalous magnetic moment favored experimentally [15], and is also consistent with the requirement of obtaining the correct Higgs mass in the minimal supersymmetric Standard Model (MSSM) [16-20].

Recently, the ATLAS Collaboration has released preliminary results of a similar search using the complete Run II data set with an integrated luminosity of $139 \mathrm{fb}^{-1}$ [21]. This analysis examines the same trilepton topologies considered in Ref. [8] using a new suite of kinematic variables designed to emulate the RJR analysis, dubbed effective RJR (eRJR). While an excess of events persists in the eRJR analysis, the significance of the excess decreases to $\sim 1 \sigma$. The limits set by this new analysis are therefore slightly more stringent compared to the original RJR search. Accommodating the updated constraints requires lowering the value of $M_{2}$ by a few $\mathrm{GeV}$, which compresses the neutralino mass spectrum while lowering the signal 
acceptance. In the following discussion we will adopt this approach, squeezing the $\chi_{2}^{0}-\chi_{1}^{0}$ mass splitting in order to reduce the LHC search's sensitivity.

Indirect searches for DM have led to the observation of a significant gamma-ray excess at the center of the Galaxy $[22,23]$. If interpreted in terms of DM annihilating mainly into bottom-quark pairs, the cross section needed to explain such an excess is close to the one necessary to explain the relic density in a freeze-out scenario [24-28]. This numerical fact has led to the expectation that the annihilation of a standard weakly interacting massive particle may be responsible for the observed Galactic Center excess (GCE). The initial excitement was subsequently contained by an analysis that demonstrated a preference for point-like sources (PSs) instead of DM as a possible source of the GCE. This suggested some unresolved astrophysical objects, most likely millisecond pulsars, as the origin of the observed excess [29,30]. However, a recent reexamination [31] of the analysis of Ref. [29] has shown that this reported preference for PSs may be due to a mismodeling of the real unknown distribution of PSs in the inner Galaxy, and calls into question any inference from Ref. [29] that the DM contribution to the GCE is small. Namely, it was found in Ref. [31] that even if one injects an artificially enhanced simulated DM signal (without any point-like sources) into the real Fermi data, the analysis pipeline of Ref. [29] still misattributes this to PSs. Therefore the DM interpretation which was previously thought to be disfavored, can again be considered as the possible origin of the GCE.

Intriguingly, DM masses of about $60 \mathrm{GeV}$ best fit the GCE, and as mentioned above, are consistent with the ones that lead to an explanation of the RJR trilepton signatures. Moreover, a recent excess in antiprotons observed by the AMS-02 experiment [32] seems to be consistent with DM annihilating into bottom-quark pairs, with a similar range of DM masses, and for annihilation cross sections consistent with the ones necessary to explain the GCE [33-37]. Such scenarios are constrained by indirect searches of dwarf spheroidal galaxies (dSph) [38], which we will briefly comment on. In view of all of the above, it seems highly relevant to investigate extensions of our previous work [7] that, without changing the main results allowing for the interpretation of a possible RJR excess in the ATLAS trilepton plus missing energy signal, could lead to a DM explanation of the GCE (as well as the antiproton excess observed by AMS-02). In Sec. II we will discuss two such extensions involving the $C P$-violating MSSM (CPVMSSM) and the next-to-minimal supersymmetric Standard Model (NMSSM), respectively. We reserve Sec. III for our conclusions.

\section{GALACTIC CENTER EXCESS IN SUPERSYMMETRY}

In our previous work [7] the proper DM relic density was obtained by resonant annihilation mediated by the Standard
Model (SM)-like Higgs boson $h$ for a lightest bino-like neutralino DM candidate $\chi$ with mass $\sim 60 \mathrm{GeV}$. The relevant Lagrangian density is given by

$$
\mathcal{L}=-g_{h \chi \chi} \chi \chi h-m_{\chi} \chi \chi+\text { H.c. }
$$

If the mass parameter and the coupling, $m_{\chi}$ and $g_{h \chi \chi}$, are real, as in the $C P$-conserving MSSM, the $\chi$ annihilation process will be $p$-wave suppressed and therefore no relevant indirect detection signal is expected. A pseudoscalar coupling of the DM candidate to a mediator is required to generate an $s$-wave annihilation cross section. Further, as has been explained in Ref. [39], even for the case of a dominantly $s$-wave annihilation cross section, due to thermal fluctuations, the matching of a resonantly enhanced annihilation cross section at the freeze-out temperature $T_{F}$ (relevant for the relic density) and $T=0$ (relevant for indirect detection) is nontrivial. In particular, in order to get the right indirect detection signal together with an observationally consistent relic density, there must be a particular relation between the mediator couplings to DM and the mediator couplings to SM particles. Actually, it is known that an explanation of the GCE within the $C P$-conserving MSSM demands masses larger than $60 \mathrm{GeV}$ [40-42].

\section{A. CPVMSSM}

If $m_{\chi}$ is complex, then an $s$-wave contribution to the $h$ mediated annihilation cross section will be generated. This happens for instance for a bino DM candidate in the presence of a nonvanishing argument for the bino mass parameter $M_{1}$. In the particular case of $\arg \left[M_{1}\right]=\pi / 2$, after absorbing the phase into a redefinition of $\chi, h$ will obtain a pseudoscalar coupling to the Majorana neutralino [proportional to $(\chi \chi-\bar{\chi} \bar{\chi})]$, and the annihilation cross section will be entirely $s$-wave. Hence, for a generic complex $m_{\chi}$ one expects potentially relevant indirect detection signals. In particular, a suitable choice of the $C P$-violating phase allows for coupling relations required for the consistency of the GCE and yields a relic density prediction consistent with observations.

Although the introduction of a nonvanishing phase to $M_{1}$ does not impact the trilepton signatures at the LHC, it does lead to the appearance of electric dipole moments (EDMs) which are strongly constrained experimentally [43]. The appearance of $C P$ violation only in the bino mass parameter, together with a sizable value of the heavy Higgs boson masses, $m_{H^{+}}>1 \mathrm{TeV}$, suppresses the two-loop contributions to the EDMs [44-47]. In this case, the main contribution to the electron EDM comes at one loop, and scales as the inverse square of the selectron masses. Values of the selectron masses of a few $\mathrm{TeV}$ are sufficient to evade the current EDM bounds. However, slepton masses at a scale of a few $\mathrm{TeV}$ will not give any significant contribution to the anomalous magnetic moment of the 
TABLE I. Benchmark values of CPVMSSM input parameters for micrOMEGAs. The squark and slepton soft masses are degenerate between generations and chiralities, and all unlisted $A$ terms are zero.

\begin{tabular}{|c|c|c|c|c|c|c|c|}
\hline Param. & Value & Param. & [GeV] & Param. & . $[\mathrm{GeV}]$ & Param. & {$[\mathrm{GeV}]$} \\
\hline $\arg \left[M_{1}\right]$ & $5.8^{\circ}$ & $\mu$ & -300 & $M_{3}$ & 3000 & $A_{t}$ & 2500 \\
\hline $\tan \beta$ & 20 & $M_{1}$ & 63.426 & $M_{\tilde{L}}$ & 3000 & $A_{b}$ & 2500 \\
\hline$M_{H^{ \pm}}$ & $1500 \mathrm{GeV}$ & $M_{2}$ & -182 & $M_{\tilde{Q}}$ & 3000 & $A_{\tau}$ & 1000 \\
\hline
\end{tabular}

muon, which then remains suppressed as compared to the experimentally favored value.

We numerically verified the above conclusions by using the CPVMSSM implementation in micrOMEGAs 5.0.8 [48,49] which uses CPsuperH $2.3[50,51]$ as the spectrum generator. We found that for

$$
m_{\chi}=\left|m_{\chi}\right| \exp (i \phi)
$$

small values of $\phi$ for a neutralino mass $\left|m_{\chi}\right| \sim 60 \mathrm{GeV}$ can lead to resonant annihilation via $h$, and one may obtain consistency with the GCE signatures and the observed relic density. The full set of CPVMSSM parameters are shown in Table I, leading to approximately the same physical spectrum, tabulated in Table II, as the one presented in Ref. [7]. We take all SUSY scalar masses of order $M_{S} \simeq 3 \mathrm{TeV}$, which suppresses all EDMs, and the value of $A_{t} \simeq 2.5 \mathrm{TeV}$, to obtain the right Higgs boson mass. Values of the other parameters are similar to the ones of the benchmark presented in Ref. [7].

The difference in the wino mass parameter, $M_{2}$, compared to the value presented in Ref. [7] is mostly due to the loop corrections to the neutralino and chargino spectrum present in the $C P$-conserving MSSM implementation of MICROMEGAs which was used in our previous work, together with a slight compression of the neutralino spectrum in order to satisfy the eRJR analysis bounds [21]. Although the exact bounds on this scenario are difficult to estimate as the acceptance rates for the eRJR

TABLE II. Benchmark mass spectrum generated from the input parameters of Table I.

\begin{tabular}{lrrrrrrr}
\hline \hline Part. & $m[\mathrm{GeV}]$ & Part. & $m[\mathrm{GeV}]$ & Part. & $m[\mathrm{GeV}]$ & Part. & $m[\mathrm{GeV}]$ \\
\hline$h$ & 125.5 & $\tilde{\chi}_{1}^{ \pm}$ & 162.7 & $\tilde{\nu}_{e}$ & 2999.3 & $\tilde{u}_{R}$ & 2999.8 \\
$H_{2}$ & 1497.9 & $\tilde{\chi}_{2}^{ \pm}$ & 331.5 & $\tilde{\nu}_{\mu}$ & 2999.3 & $\tilde{u}_{L}$ & 2999.5 \\
$H_{3}$ & 1497.9 & $\tilde{\tau}_{1}$ & 2998.4 & $\tilde{\nu}_{\tau}$ & 2999.3 & $\tilde{d}_{R}$ & 3000.1 \\
$H^{ \pm}$ & 1500.0 & $\tilde{\tau}_{2}$ & 3002.3 & $\tilde{g}$ & 3000.0 & $\tilde{d}_{L}$ & 3000.6 \\
$\tilde{\chi}_{1}^{0}$ & 62.7 & $\tilde{e}_{R}$ & 3000.3 & $\tilde{t}_{1}$ & 2945.8 & $\tilde{s}_{R}$ & 3000.1 \\
$\tilde{\chi}_{2}^{0}$ & 162.5 & $\tilde{e}_{L}$ & 3000.4 & $\tilde{t}_{2}$ & 3058.4 & $\tilde{s}_{L}$ & 3000.6 \\
$\tilde{\chi}_{3}^{0}$ & 309.7 & $\tilde{\mu}_{R}$ & 3000.3 & $\tilde{b}_{1}$ & 2997.6 & $\tilde{c}_{R}$ & 2999.8 \\
$\tilde{\chi}_{4}^{0}$ & 328.5 & $\tilde{\mu}_{L}$ & 3000.4 & $\tilde{b}_{2}$ & 3003.1 & $\tilde{c}_{L}$ & 2999.5 \\
\hline \hline
\end{tabular}

analysis are not yet public, we note that further compression of the $\chi_{2}^{0}-\chi_{1}^{0}$ mass splitting is possible by reducing $M_{2}$ without altering the DM observables in any significant way. The difference in $A_{t}$ is related to the different loop corrections used to compute the $C P$-even Higgs mass in both programs.

Further choosing the heavy Higgs boson masses to be of about $1.5 \mathrm{TeV}$ we obtain

$$
\begin{aligned}
\Omega h^{2} & =0.118, \quad \sigma_{\mathrm{SI}}^{p}=2.17 \times 10^{-12} \mathrm{pb}, \\
\sigma_{\mathrm{SI}}^{n} & =1.84 \times 10^{-12} \mathrm{pb}, \\
\left.\sigma v\right|_{v=0} & =2.53 \times 10^{-26} \mathrm{~cm}^{3} / \mathrm{s}, \quad \sigma_{\mathrm{SD}}^{p}=1.76 \times 10^{-5} \mathrm{pb}, \\
\sigma_{\mathrm{SD}}^{n} & =1.36 \times 10^{-5} \mathrm{pb} .
\end{aligned}
$$

In the above, $\Omega h^{2}$ is the DM relic density, $\left.\sigma v\right|_{v=0}$ is the annihilation cross section at $T=0$, and $\sigma_{\mathrm{SI}, \mathrm{SD}}^{n, p}$ are the DMneutron and -proton spin-independent (SI) and spin-dependent (SD) cross sections, respectively. The above numbers are in good agreement with current bounds [52-57]. As we explained in Ref. [7], similar results for the direct detection cross section are obtained for other choices of the heavy Higgs masses of the order of the $\mathrm{TeV}$ scale. Moreover, since the annihilation cross section is mediated by the SM-like Higgs boson, the zero-temperature effective annihilation cross section into $b \bar{b}$ final states is $1.5 \times 10^{-26} \mathrm{~cm}^{3} / \mathrm{s}$, in good agreement with the necessary one to explain the GCE. The remaining contributions to $\left.\sigma v\right|_{v=0}$ from other channels are approximately proportional to the SM-like Higgs branching ratios $\left(22 \%\right.$ into $W W^{*}, 8 \%$ into gluons, $6 \%$ into tau leptons, etc). For $m_{\chi} \sim 60 \mathrm{GeV}$, the main effect of these additional channels will be to slightly broaden the gamma-ray spectrum without affecting the main contribution to the GCE in a relevant way.

For the chosen values of the parameters, which include vanishing trilinear slepton mass parameters, $A_{l}=0$, the electron EDM obtained with CPSUPERH is $1.8 \times 10^{-30} e \cdot \mathrm{cm}$, which is approximately a factor of 5 lower than the current bound on this quantity [43]. Actually, for these large values of the slepton masses there is a partial cancellation between the one- and two-loop contributions that suppresses the electron EDM from its one-loop value [58]. Indeed, whereas values of the electron EDM of the order of the current experimental bound are obtained for $m_{\tilde{L}}=2 \mathrm{TeV}$, an approximate cancellation between the one- and two-loop contributions occurs for $m_{\tilde{L}} \sim 4 \mathrm{TeV}$.

\section{B. NMSSM}

An alternative scenario, which was advocated in the context of the GCE in Ref. [39], is the NMSSM. The appearance of additional $C P$-odd and $C P$-even singlets in the NMSSM allows for the presence of extra channels contributing to the resonant annihilation of DM. For a 
TABLE III. Benchmark values of NMSSM input parameters for micrOMEGAs. The squark and slepton soft masses are degenerate between generations and chiralities, and all unlisted $A$ terms are $1 \mathrm{TeV}$.

\begin{tabular}{lccccccc}
\hline \hline Param. & Value & Param. & {$[\mathrm{GeV}]$} & Param. & {$[\mathrm{GeV}]$} & Param. & {$[\mathrm{GeV}]$} \\
\hline $\tan \beta$ & 20 & $\mu_{\text {eff }}$ & -300 & $M_{3}$ & 3000 & $A_{\lambda}$ & -1260 \\
$\lambda$ & 0.15 & $M_{1}$ & 62.64 & $M_{\tilde{L}}$ & 450 & $A_{\kappa}$ & -10.8 \\
$\kappa$ & -0.55 & $M_{2}$ & -166. & $M_{\tilde{Q}}$ & 3000 & $A_{t}$ & 4000 \\
\hline \hline
\end{tabular}

$60 \mathrm{GeV}$, mostly bino DM candidate, compatibility with the GCE and relic density may be obtained via resonant annihilation mediated by both the $C P$-odd singlet $A_{1}$ (to give the relevant $s$-wave contribution at zero temperature) as well as through $h$ (to provide the dominant contribution to the finite-temperature annihilation cross section relevant for the relic density). ${ }^{1}$

One advantage of the NMSSM compared to the CPVMSSM scenario is the possibility of preserving $C P$ and hence avoiding the EDM constraints. One can then lower the slepton masses to values of order $450 \mathrm{GeV}$, leading to a sizable contribution to the muon anomalous magnetic moment, which is experimentally favored [15]. Using NMSSMTools 5.1.1 [59-64] which is the spectrum generator for the NMSSM in micrOMEGAs 5.0.8, we choose the parameters tabulated in Table III, such that, as shown in Table IV, a similar neutralino/chargino spectrum as in our previous work [7] is obtained, again with a slightly more compressed neutralino spectrum in order to take into account the new eRJR analysis constraints. These parameters are consistent with the current collider constraints [21]. Moreover, with the choice of slepton masses $M_{\tilde{L}}=450 \mathrm{GeV}$, these parameter choices also lead to consistency with the muon anomalous magnetic moment, $a_{\mu}^{\mathrm{MSSM}}=217 \times 10^{-11}$.

The values of the parameters in the singlet sector are selected to keep the lightest neutralino MSSM-like. This is achieved for $|\kappa / \lambda|>1$, for which the singlino state becomes heavier than the Higgsinos. Since the singlet $C P$-even scalar tends to be heavy in this region of parameters it does not lead to any large mixing effects on the SM-like Higgs properties. The value of $A_{\kappa}$ is chosen to obtain a lightest $C P$-odd mass consistent with the resonant annihilation condition. Finally, the value of $A_{\lambda}$ was chosen to ensure a heavy enough doublet Higgs spectrum to avoid direct LHC search constraints, and to provide significant cancellations in the direct detection cross section amplitudes $[39,65]$. We observe that the same physical spectrum and hence the same physical results can

\footnotetext{
${ }^{1}$ Details of such a mechanism may be found in Ref. [39], where it was shown that for a slightly lighter singlino-Higgsino DM candidate of mass $m_{\chi} \sim 40 \mathrm{GeV}, A_{1}$ can assist annihilation close to the $Z$ funnel, and yield consistency with the GCE and the observed relic density.
}

be obtained for a broad range of correlated values of the model parameters.

One obtains a lightest neutralino mass of the order of $60 \mathrm{GeV}$, a second lightest neutralino of about $165 \mathrm{GeV}$, and a light pseudoscalar with mass $\sim 120 \mathrm{GeV}$. The doubletlike Higgs boson masses are $m_{\mathrm{A}_{2}} \sim m_{\mathrm{H}_{2}} \sim 970 \mathrm{GeV}$, and the heavier $C P$-even singlet is $\sim 2.2 \mathrm{TeV}$. With this mass spectrum, one obtains

$$
\begin{aligned}
\Omega h^{2} & =0.119, \quad \sigma_{\mathrm{SI}}^{p}=5.57 \times 10^{-12} \mathrm{pb}, \\
\sigma_{\mathrm{SI}}^{n} & =7.18 \times 10^{-12} \mathrm{pb}, \\
\left.\sigma v\right|_{v=0} & =2.22 \times 10^{-26} \mathrm{~cm}^{3} / \mathrm{s}, \quad \sigma_{\mathrm{SD}}^{p}=1.59 \times 10^{-5} \mathrm{pb} \\
\sigma_{\mathrm{SD}}^{n} & =1.23 \times 10^{-5} \mathrm{pb} .
\end{aligned}
$$

In this scenario, since the zero-temperature annihilation cross section is mediated primarily by the light pseudoscalar $A_{1}$, the contribution of the $b \bar{b}$ channel to $\left.\sigma v\right|_{v=0}$ is about $90 \%$. Hence, again, the cross section is consistent with the one necessary to explain the GCE. As in the previous scenario, the SI and SD direct detection cross sections are in good agreement with current bounds [52-57].

We observe that, albeit small in the resonant case discussed, the mixing of the singlet pseudoscalar and the doublet pseudoscalar state is crucial for obtaining the GCE. In principle, such a mixing also leads to an increase of the production cross section of $A_{1}$ at colliders which can be searched for at the LHC. However, even though the lightest pseudoscalar has a mass of about $120 \mathrm{GeV}$, with $\operatorname{BR}\left(A_{1} \rightarrow\right.$ $b \bar{b}) \sim 90 \%$ and $\operatorname{BR}\left(A_{1} \sim \tau^{+} \tau^{-}\right) \sim 10 \%$, respectively, its effective gluon-fusion production cross section is only $\mathcal{O}(1) \mathrm{pb}$. Such a small production cross section makes it challenging to test at the LHC. The prospects for the heavy $C P$-even and $C P$-odd doublet-like Higgs bosons are more interesting. On the one hand, the decays of both $\mathrm{H}_{2}$ and $\mathrm{A}_{2}$ into many different weakly interacting states (such as charginos, neutralinos and staus) suppresses their $\tau$-pair signatures, allowing them to evade current limits. On the other hand, their branching ratio into $\tau$ pairs is still $\sim 4 \%$, which makes them potentially detectable in this channel with more luminosity. Further, their decays into charginos/ neutralinos as well as cascade decays into additional Higgs bosons may provide complimentary search handles at the LHC [66-72].

\section{CONCLUSIONS}

In this work, we have presented scenarios that lead to a simultaneous explanation of an excess in trileptons plus missing energy at the LHC, a gamma-ray excess at the center of the Galaxy, and an antiproton excess in cosmic rays. These are based on either the MSSM with $C P$ violation in the neutralino sector, or on the NMSSM with a light $C P$-odd scalar. The lightest neutralino acquires a 
TABLE IV. NMSSM Benchmark mass spectrum generated from the input parameters of Table III.

\begin{tabular}{lrrrrrrr}
\hline \hline Part. & $m[\mathrm{GeV}]$ & Part. & $m[\mathrm{GeV}]$ & Part. & $m[\mathrm{GeV}]$ & Part. & $m[\mathrm{GeV}]$ \\
\hline$h$ & 124.8 & $\tilde{\chi}_{1}^{ \pm}$ & 160.7 & $A_{1}$ & 120.8 & $\tilde{u}_{R}$ & 3100.7 \\
$H_{2}$ & 970.1 & $\tilde{\chi}_{2}^{ \pm}$ & 336.1 & $A_{2}$ & 974.6 & $\tilde{u}_{L}$ & 3100.5 \\
$H_{3}$ & 2185.5 & $\tilde{\tau}_{1}$ & 438.3 & $\tilde{\nu}_{e, \mu, \tau}$ & 445.7 & $\tilde{d}_{R}$ & 3101.0 \\
$H^{ \pm}$ & 973.4 & $\tilde{\tau}_{2}$ & 465.5 & $\tilde{g}$ & 3198.1 & $\tilde{d}_{L}$ & 3101.5 \\
$\tilde{\chi}_{1}^{0}$ & 60.7 & $\tilde{e}_{R}$ & 452.0 & $\tilde{t}_{1}$ & 2955.6 & $\tilde{s}_{R}$ & 3101.0 \\
$\tilde{\chi}_{2}^{0}$ & 160.5 & $\tilde{e}_{L}$ & 452.3 & $\tilde{t}_{2}$ & 3120.5 & $\tilde{s}_{L}$ & 3101.5 \\
$\tilde{\chi}_{3}^{0}$ & 315.9 & $\tilde{\mu}_{R}$ & 452.0 & $\tilde{b}_{1}$ & 3076.3 & $\tilde{c}_{R}$ & 3100.7 \\
$\tilde{\chi}_{4}^{0}$ & 333.2 & $\tilde{\mu}_{L}$ & 452.3 & $\tilde{b}_{2}$ & 3077.8 & $\tilde{c}_{L}$ & 3100.5 \\
\hline \hline
\end{tabular}

mass of order $60 \mathrm{GeV}$, while the second lightest neutralino and the lightest chargino have masses of order $160 \mathrm{GeV}$. While the CPVMSSM scenario may lead to an observable electron EDM, the NMSSM scenario may be tested by searches at the LHC for heavy scalars decaying to $\tau$ pairs, electroweakinos or additional scalars. The latter scenario also gives rise to values of the muon anomalous magnetic moment consistent with current observations. Both benchmarks are found to be consistent with the dSph limits set in Ref. [38].

In closing, let us emphasize that while the chargino and neutralino spectrum leading to an explanation of the LHC trilepton excess depends crucially on $M_{2}$, the DM properties are fairly independent of the value of $M_{2}$. The benchmark scenarios presented above have a neutralino spectrum that is somewhat more compressed than the one presented in Ref. [7], allowing them to satisfy the somewhat tighter constraints obtained by the eRJR analysis [21]. The exact value of $M_{2}$ required to fit the remaining LHC excess depends on the signal acceptance, and we eagerly await further analysis of the complete Run II data set. However, it should be restated that the main conclusions of this paper and of our previous work are largely independent of whether or not this excess is eventually confirmed. A low-energy electroweakino spectrum remains a tantalizing possibility of simultaneously explaining $\left(g_{\mu}-2\right)$, DM direct detection nonobservation, and, as we have demonstrated here, the GC and antiproton excesses.

\section{ACKNOWLEDGMENTS}

We thank D. Hooper for initially suggesting the idea of $C P$ violation in the DM-Higgs coupling. We additionally thank R. Leanne and T. Slatyer for useful discussions and comments. This manuscript has been authored by Fermi Research Alliance, LLC under Contract No. DE-AC0207CH11359 with the U.S. Department of Energy, Office of Science, Office of High Energy Physics. Work at University of Chicago is supported in part by U.S. Department of Energy Grant No. DE-FG02-13ER41958. Work at ANL is supported in part by the U.S. Department of Energy under Contract No. DE-AC02-06CH11357. N.R.S. and J.O. are supported by Wayne State University and by the U.S. Department of Energy under Contract No. DESC0007983.
[1] M. Aaboud et al. (ATLAS Collaboration), Search for squarks and gluinos in final states with jets and missing transverse momentum using $36 \mathrm{fb}^{-1}$ of $\sqrt{s}=13 \mathrm{TeV}$ pp collision data with the ATLAS detector, Phys. Rev. D 97, 112001 (2018).

[2] A. M. Sirunyan et al. (CMS Collaboration), Search for natural and split supersymmetry in proton-proton collisions at $\sqrt{s}=13 \mathrm{TeV}$ in final states with jets and missing transverse momentum, J. High Energy Phys. 05 (2018) 025.

[3] M. Aaboud et al. (ATLAS Collaboration), Search for supersymmetry in final states with missing transverse momentum and multiple $b$-jets in proton-proton collisions at $\sqrt{s}=13 \mathrm{TeV}$ with the ATLAS detector, J. High Energy Phys. 06 (2018) 107.

[4] M. Aaboud et al. (ATLAS Collaboration), Search for topsquark pair production in final states with one lepton, jets, and missing transverse momentum using $36 \mathrm{fb}^{-1}$ of $\sqrt{\mathrm{s}}=$ $13 \mathrm{TeV}$ pp collision data with the ATLAS detector, J. High Energy Phys. 06 (2018) 108.

[5] A. M. Sirunyan et al. (CMS Collaboration), Search for top squarks decaying via four-body or chargino-mediated modes in single-lepton final states in proton-proton collisions at $\sqrt{s}=13 \mathrm{TeV}$, J. High Energy Phys. 09 (2018) 065.

[6] A. M. Sirunyan et al. (CMS Collaboration), Searches for pair production of charginos and top squarks in final states with two oppositely charged leptons in proton-proton collisions at $\sqrt{s}=13 \mathrm{TeV}$, J. High Energy Phys. 11 (2018) 079.

[7] M. Carena, J. Osborne, N. R. Shah, and C. E. M. Wagner, Supersymmetry and LHC missing energy signals, Phys. Rev. D 98, 115010 (2018).

[8] M. Aaboud et al. (ATLAS Collaboration), Search for chargino-neutralino production using recursive jigsaw reconstruction in final states with two or three charged leptons in proton-proton collisions at $\sqrt{s}=13 \mathrm{TeV}$ with the ATLAS detector, Phys. Rev. D 98, 092012 (2018).

[9] P. Jackson, C. Rogan, and M. Santoni, Sparticles in motion: Analyzing compressed SUSY scenarios with a new method of event reconstruction, Phys. Rev. D 95, 035031 (2017).

[10] P. Jackson and C. Rogan, Recursive Jigsaw reconstruction: HEP event analysis in the presence of kinematic and combinatoric ambiguities, Phys. Rev. D 96, 112007 (2017). 
[11] M. Aaboud et al. (ATLAS Collaboration), Search for electroweak production of supersymmetric particles in final states with two or three leptons at $\sqrt{s}=13 \mathrm{TeV}$ with the ATLAS detector, Eur. Phys. J. C 78, 995 (2018).

[12] A. M. Sirunyan et al. (CMS Collaboration), Combined search for electroweak production of charginos and neutralinos in proton-proton collisions at $\sqrt{s}=13 \mathrm{TeV}$, J. High Energy Phys. 03 (2018) 160.

[13] A. Kvellestad (GAMBIT Collaboration), Global fits of the MSSM with GAMBIT, Proc. Sci. ICHEP2018 (2019) 402.

[14] P. Athron et al. (GAMBIT Collaboration), Combined collider constraints on neutralinos and charginos, Eur. Phys. J. C 79, 395 (2019).

[15] G. W. Bennett et al. (Muon g-2 Collaboration), Final report of the muon E821 anomalous magnetic moment measurement at BNL, Phys. Rev. D 73, 072003 (2006).

[16] H. Bahl, S. Heinemeyer, W. Hollik, and G. Weiglein, Reconciling EFT and hybrid calculations of the light MSSM Higgs-boson mass, Eur. Phys. J. C 78, 57 (2018).

[17] E. Bagnaschi, G. F. Giudice, P. Slavich, and A. Strumia, Higgs mass and unnatural supersymmetry, J. High Energy Phys. 09 (2014) 092.

[18] P. Draper, G. Lee, and C. E. M. Wagner, Precise estimates of the Higgs mass in heavy supersymmetry, Phys. Rev. D 89, 055023 (2014).

[19] G. Lee and C. E. M. Wagner, Higgs bosons in heavy supersymmetry with an intermediate $\mathrm{m}_{A}$, Phys. Rev. D 92, 075032 (2015).

[20] J. Pardo Vega and G. Villadoro, SusyHD: Higgs mass determination in Supersymmetry, J. High Energy Phys. 07 (2015) 159.

[21] ATLAS Collaboration, Search for chargino-neutralino production with mass splittings near the electroweak scale in three-lepton final states in $\sqrt{s}=13 \mathrm{TeV} p p$ collisions with the ATLAS detector, Technical Report No. ATLAS-CONF2019-020, CERN, Geneva, 2019.

[22] D. Hooper and L. Goodenough, Dark matter annihilation in the galactic center as seen by the Fermi gamma ray space telescope, Phys. Lett. B 697, 412 (2011).

[23] M. Ajello et al. (Fermi-LAT Collaboration), Fermi-LAT observations of high-energy $\gamma$-ray emission toward the galactic center, Astrophys. J. 819, 44 (2016).

[24] D. Hooper and T. Linden, On the origin of the gamma rays from the galactic center, Phys. Rev. D 84, 123005 (2011).

[25] K. N. Abazajian and M. Kaplinghat, Detection of a gammaray source in the galactic center consistent with extended emission from dark matter annihilation and concentrated astrophysical emission, Phys. Rev. D 86, 083511 (2012).

[26] T. Daylan, D. P. Finkbeiner, D. Hooper, T. Linden, S. K. N. Portillo, N. L. Rodd, and T. R. Slatyer, The characterization of the gamma-ray signal from the central Milky Way: A case for annihilating dark matter, Phys. Dark Universe 12, 1 (2016).

[27] P. Agrawal, B. Batell, P. J. Fox, and R. Harnik, WIMPs at the galactic center, J. Cosmol. Astropart. Phys. 05 (2015) 011.

[28] C. Karwin, S. Murgia, T. M. P. Tait, T. A. Porter, and P. Tanedo, Dark matter interpretation of the Fermi-LAT observation toward the galactic center, Phys. Rev. D 95, 103005 (2017).
[29] S. K. Lee, M. Lisanti, B. R. Safdi, T. R. Slatyer, and W. Xue, Evidence for Unresolved $\gamma$-Ray Point Sources in the Inner Galaxy, Phys. Rev. Lett. 116, 051103 (2016).

[30] R. Bartels, S. Krishnamurthy, and C. Weniger, Strong Support for the Millisecond Pulsar Origin of the Galactic Center GeV Excess, Phys. Rev. Lett. 116, 051102 (2016).

[31] R. K. Leane and T. R. Slatyer, Dark matter strikes back at the galactic center, arXiv:1904.08430.

[32] M. Aguilar et al. (AMS Collaboration), Antiproton Flux, Antiproton-to-Proton Flux Ratio, and Properties of Elementary Particle Fluxes in Primary Cosmic Rays Measured with the Alpha Magnetic Spectrometer on the International Space Station, Phys. Rev. Lett. 117, 091103 (2016).

[33] M.-Y. Cui, Q. Yuan, Y.-L. S. Tsai, and Y.-Z. Fan, Possible Dark Matter Annihilation Signal in the AMS-02 Antiproton Data, Phys. Rev. Lett. 118, 191101 (2017).

[34] A. Cuoco, M. Krämer, and M. Korsmeier, Novel Dark Matter Constraints from Antiprotons in Light of AMS-02, Phys. Rev. Lett. 118, 191102 (2017).

[35] M.-Y. Cui, X. Pan, Q. Yuan, Y.-Z. Fan, and H.-S. Zong, Revisit of cosmic ray antiprotons from dark matter annihilation with updated constraints on the background model from AMS-02 and collider data, J. Cosmol. Astropart. Phys. 06 (2018) 024.

[36] A. Cuoco, J. Heisig, M. Korsmeier, and M. Krämer, A combined dark matter study of AMS-02 antiprotons and Fermi-LAT gamma rays, Proc. Sci., EPS-HEP2017 (2017) 065.

[37] I. Cholis, T. Linden, and D. Hooper, A robust excess in the cosmic-ray antiproton spectrum: Implications for annihilating dark matter, Phys. Rev. D 99, 103026 (2019).

[38] A. Albert et al. (Fermi-LAT and DES Collaborations), Searching for dark matter annihilation in recently discovered milky way satellites with Fermi-LAT, Astrophys. J. 834, 110 (2017).

[39] C. Cheung, M. Papucci, D. Sanford, N. R. Shah, and K. M. Zurek, NMSSM interpretation of the galactic center excess, Phys. Rev. D 90, 075011 (2014).

[40] A. Achterberg, S. Amoroso, S. Caron, L. Hendriks, R. R. de Austri, and C. Weniger, A description of the galactic center excess in the minimal supersymmetric Standard Model, J. Cosmol. Astropart. Phys. 08 (2015) 006.

[41] K. Freese, A. Lopez, N. R. Shah, and B. Shakya, MSSM Afunnel and the galactic center excess: Prospects for the LHC and direct detection experiments, J. High Energy Phys. 04 (2016) 059.

[42] T. Gherghetta, B. von Harling, A. D. Medina, M. A. Schmidt, and T. Trott, SUSY implications from WIMP annihilation into scalars at the Galactic Center, Phys. Rev. D 91, 105004 (2015).

[43] V. Andreev et al. (ACME Collaboration), Improved limit on the electric dipole moment of the electron, Nature (London) 562, 355 (2018).

[44] D. Chang, W.-F. Chang, and W.-Y. Keung, Electric dipole moment in the split supersymmetry models, Phys. Rev. D 71, 076006 (2005).

[45] G. F. Giudice and A. Romanino, Electric dipole moments in split supersymmetry, Phys. Lett. B 634, 307 (2006). 
[46] J. R. Ellis, J. S. Lee, and A. Pilaftsis, Electric dipole moments in the MSSM reloaded, J. High Energy Phys. 10 (2008) 049.

[47] Y. Li, S. Profumo, and M. Ramsey-Musolf, Bino-driven electroweak baryogenesis with highly suppressed electric dipole moments, Phys. Lett. B 673, 95 (2009).

[48] G. Belanger, F. Boudjema, A. Pukhov, and A. Semenov, micrOMEGAs3: A program for calculating dark matter observables, Comput. Phys. Commun. 185, 960 (2014).

[49] G. Bélanger, F. Boudjema, A. Goudelis, A. Pukhov, and B. Zaldivar, micrOMEGAs5.0: Freeze-in, Comput. Phys. Commun. 231, 173 (2018).

[50] J. S. Lee, A. Pilaftsis, M. Carena, S. Y. Choi, M. Drees, J. Ellis, and C.E.M. Wagner, CPsuperH: A computational tool for Higgs phenomenology in the minimal supersymmetric standard model with explicit $C P$ violation, Comput. Phys. Commun. 156, 283 (2004).

[51] J. S. Lee, M. Carena, J. Ellis, A. Pilaftsis, and C. E. M. Wagner, CPsuperH2.3: An updated tool for phenomenology in the MSSM with explicit $C P$ violation, Comput. Phys. Commun. 184, 1220 (2013).

[52] E. Aprile et al. (XENON Collaboration), First Dark Matter Search Results from the XENON1T Experiment, Phys. Rev. Lett. 119, 181301 (2017).

[53] E. Aprile et al. (XENON Collaboration), Dark Matter Search Results from a One Tonne $\times$ Year Exposure of XENON1T, Phys. Rev. Lett. 121, 111302 (2018).

[54] D. S. Akerib et al. (LUX Collaboration), Results from a Search for Dark Matter in the Complete LUX Exposure, Phys. Rev. Lett. 118, 021303 (2017).

[55] D. S. Akerib et al. (LUX Collaboration), Limits on SpinDependent WIMP-Nucleon Cross Section Obtained from the Complete LUX Exposure, Phys. Rev. Lett. 118, 251302 (2017).

[56] C. Amole et al. (PICO Collaboration), Dark Matter Search Results from the PICO-60 $\mathrm{C}_{3} \mathrm{~F}_{8}$ Bubble Chamber, Phys. Rev. Lett. 118, 251301 (2017).

[57] C. Amole et al. (PICO Collaboration), Dark Matter Search Results from the Complete Exposure of the PICO-60 $\mathrm{C}_{3} \mathrm{~F}_{8}$ Bubble Chamber, Phys. Rev. D 100, 022001 (2019).

[58] T. Ibrahim and P. Nath, $C P$ violation from Standard Model to strings, Rev. Mod. Phys. 80, 577 (2008).

[59] G. Belanger, F. Boudjema, C. Hugonie, A. Pukhov, and A. Semenov, Relic density of dark matter in the NMSSM, J. Cosmol. Astropart. Phys. 09 (2005) 001.
[60] F. Domingo and U. Ellwanger, Updated constraints from $B$ physics on the MSSM and the NMSSM, J. High Energy Phys. 12 (2007) 090.

[61] A. Djouadi, J. Kalinowski, and M. Spira, HDECAY: A program for Higgs boson decays in the standard model and its supersymmetric extension, Comput. Phys. Commun. 108, 56 (1998).

[62] G. Degrassi and P. Slavich, On the radiative corrections to the neutral Higgs boson masses in the NMSSM, Nucl. Phys. B825, 119 (2010).

[63] U. Ellwanger and C. Hugonie, NMHDECAY 2.0: An updated program for sparticle masses, Higgs masses, couplings and decay widths in the NMSSM, Comput. Phys. Commun. 175, 290 (2006).

[64] U. Ellwanger, J. F. Gunion, and C. Hugonie, NMHDECAY: A Fortran code for the Higgs masses, couplings and decay widths in the NMSSM, J. High Energy Phys. 02 (2005) 066.

[65] P. Huang and C. E. M. Wagner, Blind spots for neutralino dark matter in the MSSM with an intermediate $m_{A}$, Phys. Rev. D 90, 015018 (2014).

[66] S. Baum, K. Freese, N. R. Shah, and B. Shakya, NMSSM Higgs boson search strategies at the LHC and the monoHiggs signature in particular, Phys. Rev. D 95, 115036 (2017).

[67] S. Baum and N.R. Shah, Two Higgs doublets and a complex singlet: Disentangling the decay topologies and associated phenomenology, J. High Energy Phys. 12 (2018) 044.

[68] S. Baum, N. R. Shah, and K. Freese, The NMSSM is within reach of the LHC: Mass correlations \& decay signatures, J. High Energy Phys. 04 (2019) 011

[69] S. Baum and N. R. Shah, Benchmark suggestions for resonant double Higgs production at the LHC for extended Higgs sectors, arXiv:1904.10810.

[70] S. Gori, Z. Liu, and B. Shakya, Heavy Higgs as a portal to the supersymmetric electroweak sector, J. High Energy Phys. 04 (2019) 049.

[71] A. Adhikary, S. Banerjee, R. K. Barman, and B. Bhattacherjee, Resonant heavy Higgs searches at the HL-LHC, arXiv: 1812.05640.

[72] P. Basler, S. Dawson, C. Englert, and M. Mühlleitner, Showcasing $\mathrm{HH}$ production: Benchmarks for the LHC and HL-LHC, Phys. Rev. D 99, 055048 (2019). 